\title{
Squeezing MOND into a Cosmological Scenario
}

\author{
Arthur Lue and Glenn D. Starkman \\ Center for Education and Research in Cosmology and Astrophysics, Department of Physics, Case Western Reserve University, \\ Cleveland, Ohio 44106-7079, USA \\ and CERN Theory Division, CH-1211 Geneva 23, Switzerland
}

(Received 16 October 2003; published 2 April 2004)

\begin{abstract}
Explaining the effects of dark matter using modified gravitational dynamics (MOND) has for decades been both an intriguing and controversial possibility. By insisting that the gravitational interaction that accounts for the Newtonian force also drives cosmic expansion, one may kinematically identify which cosmologies are compatible with MOND, without explicit reference to the underlying theory so long as the theory obeys Birkhoff's law. We find that the critical acceleration $a_{0}$ must have a slight source-mass dependence $\left(a_{0} \sim M^{1 / 3}\right)$ and that MOND cosmologies are naturally compatible with observed late-time expansion history. However, cosmologies that can produce enough density perturbations to account for structure formation are contrived and fine tuned. Even then, they may be marginally ruled out by evidence of early $(z \sim 20)$ reionization.
\end{abstract}

DOI: 10.1103/PhysRevLett.92.131102

That approximately $90 \%$ of the matter in the Universe is composed of as yet unspecified material is unsettling, especially as an increasingly coherent picture of cosmology emerges. While there are well-motivated candidates for dark matter, its sole purpose is to supplement the mass in astrophysical and cosmological settings where the accounting of visible matter falls short of gravitational requirements. Replacing dark matter with a modified law of gravity (as encoded in the paradigm of Modified Newtonian Dynamics, MOND) has been for decades both an intriguing and a controversial alternative [1,2].

The dark-matter paradigm has made many successful contributions to the standard cosmological model. In contrast, MOND is largely inarticulate concerning cosmology: it is a paradigm designed to address galaxy rotation curves, there exists no satisfactory underlying theory, and there is some difficulty in incorporating it into a believable cosmological scenario [3-7].

In this Letter, we reexamine the possibility of folding MOND into a cosmological model under the premise that the same gravitational interactions that manifest themselves in a modified Newtonian force are also responsible for cosmological evolution. In a previous paper [8] with Scoccimarro, we showed how one may kinematically derive a unique Schwartzchild-like metric for a modified-gravity theory from a specified homogeneous cosmology. There too, the presumption exploited was that cosmology is driven exclusively by gravitational selfinteractions of the constituent matter, rather than by some unknown energy momentum such as dark energy. This correspondence between the metric and cosmology can be made without reference to the fundamental modifiedgravity theory, assuming only that the underlying theory respects Birkhoff's law. The procedure is simply the generalization to a full metric theory of the classic derivation of the Friedmann equation from the Newtonian force law.
PACS numbers: $04.50 .+\mathrm{h}, 98.80 .-\mathrm{k}$

We apply the same technique here to ascertain which cosmologies are compatible with MOND, allowing us to identify a full Schwartzchild-like metric and providing a self-consistent framework to perform calculations of interest in MOND cosmology. We begin by briefly reviewing the prescription for the full metric consistent with homogeneous cosmologies and then apply that prescription to determine both the Schwartzchild-like metric and the modified Friedmann equation of MOND. We then examine the class of cosmologies consistent with the MOND force law and reveal that there are potentially insurmountable difficulties that arise when incorporating MOND into a consistent cosmological scenario.

Let us quickly review the technique developed in Ref. [8] where one infers the Schwarzschild metric from an arbitrary cosmology, to see how one might apply it in reverse and devise a cosmology consistent with Modified Newtonian Dynamics. Consider a homogeneous cosmology described by the line element

$$
d s^{2}=d t^{2}-a^{2}(t) \delta_{i j} d x^{i} d x^{j}
$$

with some specified scale-factor evolution, $a(t)$. If this universe is matter dominated [i.e., filled by homogeneous matter of density $\rho(t) \sim a^{-3}$ ], one is faced with two possibilities. Einstein gravity may be correct and then cosmology is driven by some unseen additional energymomentum components - dark matter and dark energy. Alternatively, the matter we see may be the only energy momentum, but then gravitational dynamics must be altered in a specific way to achieve the observed cosmic expansion history, $a(t)$. We follow the latter possibility.

It is convenient to represent the given scale factor $a(t)$ as the solution to some alternative Friedmann equation:

$$
\dot{a}^{2} / a^{2}=H_{0}^{2} g(x),
$$

where $x=\frac{8}{3} \pi G \rho / H_{0}^{2}$ is a dimensionless parameter, $G$ is 
Newton's constant, and $H_{0}$ is today's Hubble scale. The function $g(x)$ is determined by the given $a(t)$. If one requires that the fundamental gravitational theory respects Birkhoff's law, then one can uniquely determine the metric of a spherically symmetric source [8]. That metric is described by the line element

$$
d s^{2}=g_{00}(r) d t^{2}-g_{r r}(r) d r^{2}-r^{2} d \Omega,
$$

with

$$
g_{00}(r)=g_{r r}^{-1}=1-r^{2} H_{0}^{2} g\left(r_{g} / r^{3} H_{0}^{2}\right) .
$$

Here $r_{g}=2 G M$ is the usual Schwarzschild radius of a matter source of mass $M$. Note that the form of the metric components is completely determined by $a(t)$, and, in particular, that $r_{g}$ and $r$ can only appear in the metric in a specific combination. This point will be important when we consider how to apply this connection between cosmology and the Schwartzchild-like metric to MOND.

In MOND [1] the gravitational acceleration exerted by a body of mass $M$ obeys the relationship:

$$
a= \begin{cases}-\frac{1}{2} \frac{d g_{00}}{d r}=-G M r^{-2} & |a|>a_{0}, \\ \sim-r^{-1} & |a|<a_{0},\end{cases}
$$

for some critical acceleration, $a_{0}$. If we insist on a form for modified gravity which is compatible with a homogeneous cosmology and Birkhoff's law, its Schwartzchildlike metric must be of the form Eq. (4). The form for $g(x)$ compatible with Eq. (5) is

$$
g(x)= \begin{cases}x+c_{1} x^{2 / 3} & \text { Einstein }\left(x>x_{c}\right), \\ \beta x^{2 / 3} \ln x+c_{2} x^{2 / 3} & \text { MOND }\left(x<x_{c}\right),\end{cases}
$$

for some constant parameters, $\beta, c_{1}$, and $c_{2}$, yielding

$$
a= \begin{cases}-\frac{1}{2} \frac{r_{g}}{r^{2}}, & |a|>a_{0}, \\ -\frac{3 \beta}{2} \frac{\left.r_{g} H_{0}\right)^{2 / 3}}{r}, & |a|<a_{0},\end{cases}
$$

where the critical MOND acceleration, $a_{0}$, is

$$
a_{0}=H_{0}\left[9 \beta^{2}\left(r_{g} H_{0}\right)^{1 / 3}\right] .
$$

Observationally, we choose $\beta \approx 15$ so that for source masses the size of large galaxies $\left(M \sim 10^{11} M_{\odot}\right)$, the critical acceleration is $a_{0} \approx \frac{1}{6} H_{0}$, corresponding to $x_{c} \approx$ $7 \times 10^{4}$. To ensure that $g(x)$ is continuous across the transition at $a=a_{0}$,

$$
c_{1}=c_{2}+3 \beta[\ln (3 \beta)-1] .
$$

The remaining constant represents an arbitrary choice in zero-point energy for the Newtonian potential. Although $c_{1}$ and $c_{2}$ do not affect the Newtonian acceleration, they simulate curvature-type terms in the Friedmann equation despite the spatially flat cosmology [see Eq. (1)]. However, the resulting change in $g_{r r}$ [see Eq. (4)] causes only immeasurably small effects on gravitational lensing and the post-Newtonian parameter, $\gamma$.

The form Eq. (7) for the MOND gravitational acceleration is slightly different than that typically considered, where $a_{0}=H_{0} / 6$ is a universal constant. Compatibility with a homogeneous cosmology compels us to give $a_{0}$ a weak dependence on the source mass, $a_{0} \sim M^{1 / 3}$. This dependence is not a serious amendment to the MOND paradigm; indeed a well-motivated mass dependence may actually benefit the modified-gravity scenario. Galaxy clusters appear to stray from the original MOND parametrization [2], such that uncomfortably large light-to-mass ratios are necessary to bring objects of mass scales $\mathcal{O}\left(10^{14} M_{\odot}\right)$ into accord with MOND. In our prescription, Eq. (7), objects more massive than galaxies would exhibit effective dark-matter halos somewhat heavier than those predicted by traditional MOND, consistent with what seems to be required by observations.

Let us summarize. By requiring that the underlying fundamental gravity theory that provides MOND is compatible with both homogeneous cosmologies and Birkhoff's law, we may construct the function $g(x)$ found in Eq. (6). This determines both the modified Friedmann equation, Eq. (2), and the full Schwartzchild-like metric of a spherical mass source, Eq. (4), avoiding explicit reference to the details of the fundamental theory. With these two governing relationships, we may now articulate a whole host of important properties of cosmological interest. For example, we may compute modifications of planetary ephemeris, gravitational lensing, growth of density perturbations (both linear and nonlinear), and the late-time integrated Sachs-Wolfe (ISW) effect on the cosmic microwave background. Details of these calculations for arbitrary $g(x)$ are given in Ref. [8]. Here we focus particularly on accommodating late-time acceleration into MOND, and on the growth of fluctuations.

Beginning with a function $g(x)$ given by Eq. (6) and consistent with MOND Newtonian accelerations, Eq. (7), one can articulate a MOND cosmology. The quantity $x$ that appears in the modified Friedmann equation, Eq. (2), may be interpreted as $\Omega_{b}(t)$ assuming that the matter content in the Universe is dominated by baryons. (At earlier times one would require some MOND description of the self-gravity of radiation.) One can then immediately associate $x$ with a redshift using the relationship $x=(1+z)^{3} \Omega_{b}^{\text {today }}$. Let us investigate the cosmology in stages, beginning with the Einstein, large- $x$, stage.

The transition from Einstein to MOND takes place in galaxies at $x \sim 7 \times 10^{4}$, or correspondingly, taking $\Omega_{b}^{\text {today }} \approx 0.04$ [9], cosmologically at a redshift $z \sim 120$. Thus, cosmology at redshifts $z \gtrsim 120$ follows the ordinary Friedmann equation. But if the matter content of the Universe is solely baryonic, then matter-radiation equality occurs at $z \sim 600$. Recombination still occurs at $z \sim$ 1100 , before instead of after radiation-matter equality as in conventional dark-matter cosmology. This observation corroborates prior work regarding how MOND affects the cosmic microwave background (CMB) $[10,11]$. The acoustic oscillations that appear in the CMB anisotropy must be driven in an almost purely baryonic scenario. This prior work claims that MOND not only survives this 
drastic discrepancy from the standard cosmological model, but that some ratios of CMB peak heights indeed favor MOND. Since our prescription applies strictly only during the matter-dominated epoch, it has nothing to contribute to the understanding of MOND physics at the epoch of last scattering, although it can be used to make predictions about the late-time integrated Sachs-Wolfe (ISW) effect.

Looking at the form of Eq. (6), it is clear one cannot extend MOND force law to arbitrarily small $x$, or density. Eventually, the Hubble parameter, $H=\dot{a} / a$ vanishes and the Universe recollapses, regardless of the choice one makes for $c_{2}$. The result is intuitive if one imagines cosmology as evolving classically on the Newtonian potential at some fixed energy dictated by $c_{2}$. The scale factor, $a$, is proportional to the position of a test particle on that potential. The MOND part of the potential is logarithmic, implying that every comoving trajectory eventually has a turning point for any choice of initial energy (i.e., choice of $c_{2}$ ).

Thus, there must be a sufficiently small $x$ where MOND behavior ceases to predominate. We are guided here by the data which teach us that: (i) Our Hubble expansion rate is currently $H \equiv H_{0} \simeq 70 \mathrm{~km} / \mathrm{s}$. (A value $20 \%$ or $30 \%$ smaller than this would not change these arguments materially.) (ii) We are currently undergoing acceleration in our cosmic expansion [12,13] with $\ddot{a} / a \sim H_{0}^{2}$. (iii) The expansion before $z \sim 1.7$ was decelerating [14].

We can accommodate these considerations by modifying $g(x)$ of Eq. (6) in the following way:

$$
g(x)= \begin{cases}x+3 \beta x^{2 / 3}[\ln (3 \beta)-1], & x \gtrsim(3 \beta)^{3}, \\ \beta x^{2 / 3}[\ln (1+x)], & 0.1 \lesssim x \lesssim(3 \beta)^{3}, \\ \Omega_{\Lambda}, & x \lesssim 0.1,\end{cases}
$$

where $g(x)=\Omega_{\Lambda} \approx 0.7$ is equivalent to a cosmological constant. Figure 1 shows these different regimes and a possible smooth interpolation. It is interesting that such a simple modification may be accommodated. If $\beta$ were an order-of-magnitude larger or smaller, one could not extend the MOND regime all the way to the decelerationacceleration transition and still be able to maintain both $H \sim H_{0}$ as well as $\ddot{a} / a \sim H_{0}^{2}$.

Equations (2) and (10) represent the full modified Friedmann equation from matter-radiation equality to the present time, including the onset of today's cosmic acceleration. We may now proceed to compute the growth of linear perturbations in this cosmology. Such a computation is important because we require sufficient density perturbation growth to seed the observed structure in the Universe. The evolution of linear density perturbations for the class of theories under consideration takes a simple closed form. Take a uniform overdensity in a localized spherical region such that

$$
\rho(t)=\bar{\rho}(t)[1+\delta(t)]
$$

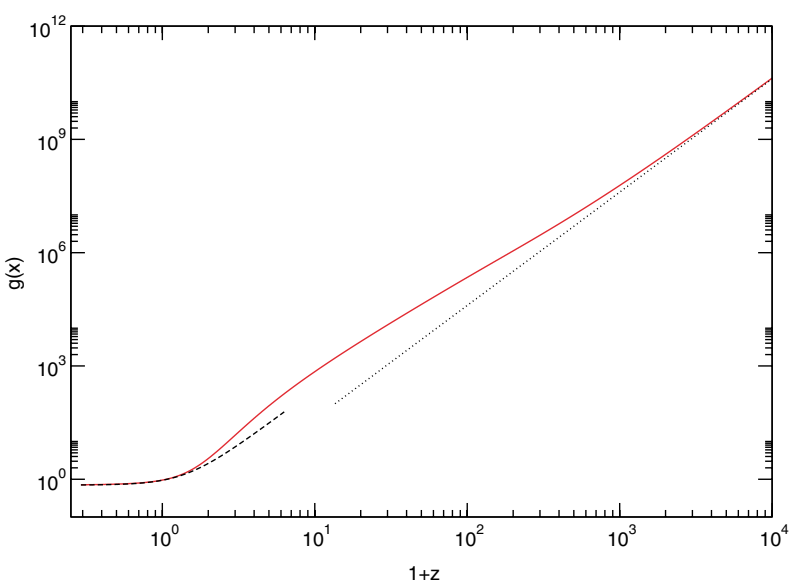

FIG. 1 (color online). The function $g(x)$ versus $1+z$ where $x=(1+z)^{3} \Omega_{b}^{\text {today }}$. The dotted line is $g(x)=x$, the asymptotic Einstein behavior at high redshifts. The dashed line is $g(x)=$ $\Omega_{\Lambda}+6 x$, which reproduces the late-time expansion history of a standard dark-matter model. The interpolating $x^{2 / 3} \ln (1+x)$ dependence is the MOND regime.

where $\bar{\rho}$ is the background matter density that follows cosmological evolution. Parametrizing time evolution using $x=8 \pi G \bar{\rho} / 3 H_{0}^{2}$, the growing perturbation mode $\delta(x)$ goes as [8] (see also [15]):

$$
\delta(x)=\frac{5 A}{6} g^{1 / 2}(x) \int_{x}^{\infty} \frac{d y}{y^{1 / 3} g^{3 / 2}(y)},
$$

where $A$ is an overall normalization. Because matter is predominantly baryons, the normalization is fixed by requiring that perturbations are restricted to be $\delta \sim$ few $\times 10^{-5}$ at recombination. Figure 2 depicts the evolution of $\delta(x)$ for a smooth interpolation of Eq. (10).

In pure matter-domination for Einstein FriedmannRobertson-Walker (FRW), $\delta=A x^{-1 / 3}$, or in other words, $\delta$ grows like the scale factor $a(t)$. Even if growth were as

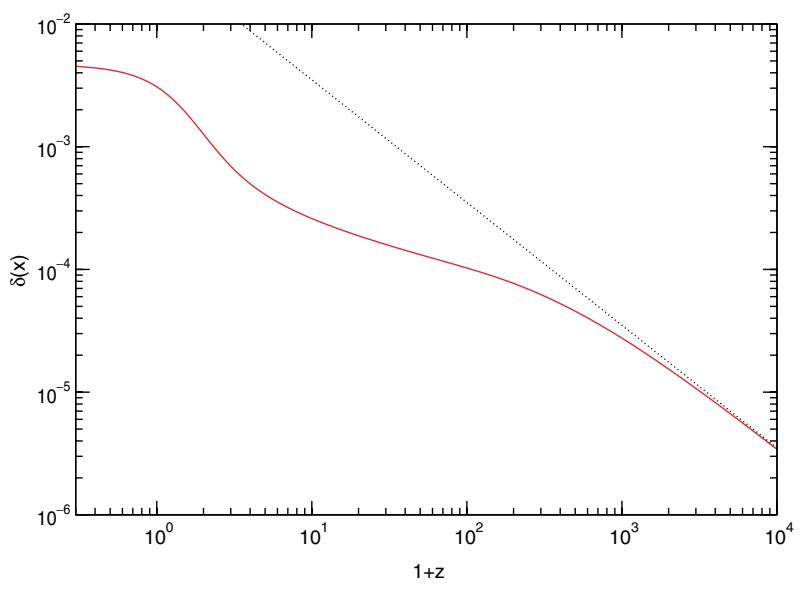

FIG. 2 (color online). Baryon density perturbation, $\delta(x)$ versus $1+z$ for $g(x)$ as in Fig. 1, normalized to the CMB anisotropy amplitude at recombination. The dotted line represents the growth in $\delta(x)$ if it were to follow Einstein gravity. 
large as this, given the normalization required at recombination, the growth of density perturbations would be insufficient to account for the observed structure formation. Moreover, in each of the three regimes in Eq. (10), growth is slower than that given benchmark, $\delta<a(t)$. In the early Einstein phase and during the MOND phase, scale-factor evolution looks as if it were curvature dominated. Growth must take place before the end of the MOND phase.

It may seem counterintuitive that growth is suppressed during the MOND regime, given that the self-gravitation of overdensities is enhanced. But the same stronger gravity also drives a faster cosmology, which in turn suppresses perturbation growth. Ultimately, this latter effect wins out. This poses a significant difficulty for cosmological incarnations of MOND.

There is a way to avoid this difficulty, but a specially tailored force law is required. Galaxy rotation curves only require that MOND be valid up to radii $r \sim 70 \mathrm{kpc}$ for galaxy masses $M \sim 10^{11} M_{\odot}[16,17]$. This distance and mass scale corresponds to $x \sim 600$ or, in MOND cosmology, to a redshift $z \sim 25$. If a recent observation of a Gunn-Peterson trough [18] signals that galaxies formed near $z \sim 6$ (or $x \sim 14$ ), then there is a narrow window in $x$, between 14 and 600, where little is known observationally and where one can carefully manipulate $g(x)$ to achieve sufficient growth in density perturbations to create galaxies, yet maintain the MOND paradigm.

To achieve the required growth, somewhere in this range of $x$, the function $g(x)$ must dip very close to zero and then rise again above $\mathcal{O}(1)$ to accommodate supernova type IA (SNIA) constraints on contemporary expansion history. From Eq. (12) one sees that near a minimum where $g(x)=g\left(x_{0}\right)+\frac{1}{2} g^{\prime \prime}\left(x_{0}\right)\left(x-x_{0}\right)^{2}$

$$
\delta \sim A x_{0}^{-1 / 3}\left[g\left(x_{0}\right) g^{\prime \prime}\left(x_{0}\right)\right]^{-1 / 2} .
$$

When $g\left(x_{0}\right)$ is close to zero, arbitrarily large growth in $\delta(x)$ can occur. The cosmology in this regime loiters, the expansion almost stops and near this critical unstable point in the potential, small variations in density amplify. For a $M \sim 10^{11} M_{\odot}$ source-mass, this fine-tuned dip in $g(x)$ corresponds to Newtonian gravity becoming repulsive in a region $r \sim 70 \rightarrow 300 \mathrm{kpc}$ to generate the large perturbations and then becoming attractive again before $r \sim 600 \mathrm{kpc}$ to account for today's cosmology.

But even this possible resolution is a tenuous one. Wilkinson Microwave Anisotropy Probe (WMAP) observations of the CMB suggests that reionization starts as early as $z \approx 20_{-9}^{+10}$ [9], and that growth in perturbations must occur before that redshift. The window for a possible excursion in $g(x)$ then becomes exceedingly small, casting doubt that a MOND cosmology can viably create the Universe we see today.

In this Letter, we provided a self-consistent framework where we could assess which cosmologies were compat- ible with Modified Newtonian Dynamics (MOND), exploiting techniques developed in prior work [8]. We found that in order for MOND to exhibit homogeneous cosmologies, the critical MOND acceleration $a_{0}$ must have a slight source-mass dependence $\left(a_{0} \sim M^{1 / 3}\right)$.

With that mild amendment, we found that MOND cosmologies are naturally compatible with observed late-time expansion histories and cosmic acceleration. However, those natural cosmologies cannot produce enough growth of density perturbations to account for structure formation. One may circumvent this difficulty by envisioning a loitering phase, arising from a drastic weakening of gravity at a selected value of $x$, chosen to correspond to part of redshift history where little is known $(z \sim 6 \rightarrow 25)$. These cosmologies are contrived and fine tuned, and may even be marginally ruled out by evidence of early $(z \sim 20)$ reionization. Such machinations cast doubt on the possibility that MOND cosmology led to the Universe we observe today.

The authors thank S. McGaugh, R. Scoccimarro, and J.-P. Uzan for helpful communications and insights and the CERN Theory Division for their hospitality. This work is sponsored by DOE Grant No. DEFG0295ER40898, the CWRU Office of the Provost, and CERN.

[1] M. Milgrom, Astrophys. J. 270, 365 (1983).

[2] R. H. Sanders and S. S. McGaugh, Annu. Rev. Astron. Astrophys. 40, 263 (2002).

[3] J. E. Felten, Astrophys. J. 286, 3 (1984).

[4] S. McGaugh, astro-ph/9812327.

[5] R. H. Sanders, Astrophys. J. 560, 1 (2001).

[6] D. Scott et al., astro-ph/0104435.

[7] A. Nusser, Mon. Not. R. Astron. Soc. 331, 909 (2002).

[8] A. Lue, R. Scoccimarro, and G. Starkman, Phys. Rev. D 69, 044005 (2004).

[9] C. L. Bennett et al., Astrophys. J. Suppl. Ser. 148, 1 (2003).

[10] S. S. McGaugh, Astrophys. J. 523, L99 (1999).

[11] S. S. McGaugh, Astrophys. J. 541, L33 (2000).

[12] Supernova Cosmology Project Collaboration, S. Perlmutter et al., Astrophys. J. 517, 565 (1999).

[13] Supernova Search Team Collaboration, A. G. Riess et al., Astron. J. 116, 1009 (1998).

[14] Supernova Search Team Collaboration, N. Benitez et al., Astrophys. J. 577, L1 (2002)

[15] T. Multamaki, E. Gaztañaga, and M. Manera, Mon. Not. R. Astron. Soc. 344, 761 (2003).

[16] R. H. Sanders, Astrophys. J. 473, 117 (1996).

[17] W. J. G. de Blok and S. S. McGaugh, Astrophys. J. 508, 132 (1998).

[18] SDSS Collaboration, R. H. Becker et al., Astron. J. 122, 2850 (2001). 